\title{
A Brief Discussion on Higgs Boson
}

\author{
D. Banerjee", P. Ghosh', S. Sahoo, ${ }^{2, *}$ \\ ${ }^{1}$ Department of Applied Physics and Ballistics, Fakir Mohan University, Balasore, Odisha, India \\ ${ }^{2}$ Department of Physics, National Institute of Technology, Durgapur, West Bengal, India \\ *Corresponding Author: sukadevsahoo@yahoo.com
}

Copyright (C) 2014 Horizon Research Publishing All rights reserved.

\begin{abstract}
The Standard Model (SM) of elementary particles allows the generation of particle mass with the help of Higgs mechanism and preserves the fundamental symmetries of the theory. The quantum of Higgs field is the Higgs boson, which was the missing piece of the SM till July 2012. It is an important particle because it is responsible for the Higgs mechanism by which all particles acquire mass. It has some unique properties which give a special status to it in the table of elementary particles of the SM. In this article, we have briefly discussed about the Higgs boson.
\end{abstract}

Keywords Higgs Boson, Standard Model, Spontaneous Symmetry Breaking, Higgs Mechanism

\section{The Standard Model and Introduction of Inertial Mass}

The Standard Model (SM) [1-6] is a versatile and elegant model which describes three of the four fundamental forces (electromagnetic, weak and strong force) in a unified framework. The gravitational force is not incorporated in the model, so the SM is also popularly known as three-fourth of everything [4]. To date, almost all experimental tests of the three forces described by the SM have agreed with its predictions with a high degree of accuracy. This model gives a mathematical formulation of the origin of mass of elementary particles by introducing a new idea, which is now known as the Higgs mechanism [7].

In the early 1960s physicists Sheldon Glashow, Stephen Weinberg and Abdus Salam attempted to unify the weak and electromagnetic forces [8-10]. From their mathematical calculations it was found that force carriers for the weak interaction were massless. This claim was consistent with the symmetry of the theory, but it was inconsistent with the experimental observation that the weak force was short range $[4,11]$. The above problem was resolved with the introduction of the Higgs mechanism. The Higgs mechanism breaks the electroweak symmetry, $\mathrm{SU}(2)_{\mathrm{L}} \times \mathrm{U}(1)_{\mathrm{Y}}$ where $\mathrm{SU}(2)_{\mathrm{L}}$ is the weak isospin gauge group and $\mathrm{U}(1)_{\mathrm{Y}}$ is the hypercharge gauge group. This symmetry breaking gives mass to $\mathrm{Z}^{0}$ and $\mathrm{W}^{ \pm}$bosons. The basic idea was that just after the big bang all particles were massless. As time passed on, the universe cooled and its temperature fell below a critical value, an invisible field called the Higgs field permeated all space. This Higgs field is responsible for providing mass to all elementary particles. The particle associated with the Higgs field is named as Higgs boson. The Higgs field is homogeneous everywhere in the space irrespective of any condition. Initially all the matter particles were massless. When these particles moved through the Higgs field, they experienced a certain amount of resistance or drag exerted by the field. This drag provided inertial mass to them. In 1964, F. Englert and R. Brout were the first to give the idea of a field that interacts with fundamental particles and give them mass [12]. In the same year Peter Higgs pointed out the potential existence of the Higgs boson first. In that year also G. S. Guralinik, C. R. Hagen and T. W. B. Kibble described a more comprehensive exposition of the Higgs mechanism.

\section{Spontaneous Symmetry Breaking and Higgs Mechanism}

A symmetry of an object (system) is an operation or a transformation $\left(S \leftrightarrow S^{\prime}\right)$ under which the object (system) remains invariant, i.e., object remains same before and after the transformation. For example, a square is invariant under reflection about a diagonal. So reflection about a diagonal is a symmetry of a square. A candle is symmetric about a vertical axis because it can be rotated about that axis without changing in appearance or any other feature; it is also symmetric with respect to reflection in a mirror. Nature loves symmetry. Each system in nature wants to be in its ground state. In particle physics each particle may be regarded as an individual system and wish to be in its respective ground state. Similarly, the gauge boson near a Higgs field wants to be in its lowest energy state which breaks at least one of the symmetries involved in the theory.

According to the gauge theory all the elementary particles and force carriers must be massless to preserve the local symmetry of Electroweak (EW) theory. However, for the weak interaction this is not the case. The $\mathrm{W}^{ \pm}$and $\mathrm{Z}^{0}$ particles have masses 80.4 and $91.2 \mathrm{GeV}$ respectively. On the other hand, the force carriers for the strong force, the 
gluon and the force carrier for the electromagnetic force, the photon, are massless. The $\mathrm{W}^{ \pm}$and $\mathrm{Z}^{0}$ bosons break the symmetry of the zero mass particles. The completion of the SM required a way of including massive force carriers without breaking the symmetries. It was found that via spontaneous symmetry breaking this could be achieved in the Higgs mechanism. The core of the SM took its present form nearly three decades ago, as a $\mathrm{SU}(3)_{\mathrm{C}} \times \mathrm{SU}(2)_{\mathrm{L}} \times \mathrm{U}(1)_{\mathrm{Y}}$ gauge theory. The $\mathrm{SU}(3)_{\mathrm{C}}$ group describes the strong interaction, also known as 'colour' force. The electroweak sector has an underlying $\mathrm{SU}(2)_{\mathrm{L}} \times \mathrm{U}(1)_{\mathrm{Y}}$ symmetry. Due to Higgs effect the weak force carriers acquired mass which breaks $\mathrm{SU}(2)_{\mathrm{L}} \times \mathrm{U}(1)_{\mathrm{Y}}$ symmetry in random way. This phenomenon of random breaking of symmetry is known as Spontaneous Symmetry Breaking (SSB) [3,13-14]. Spontaneous symmetry breaking is also defined as a spontaneous process by which a system in a symmetrical state ends up in an asymmetrical state. It thus describes systems where the equations of motion or the Lagrangian obey certain symmetries, but the lowest energy states do not exhibit that symmetry. Let us consider the bottom of an empty wine bottle, a symmetrical upward dome with a trough. If a ball is kept in a particular position at the peak of the dome, the circumferences become symmetrical with respect to rotating the wine bottle. If the bottle suddenly moves into the trough, a point of lowest energy, it spontaneously breaks this symmetry. Now the bottle and ball individually have symmetry but the system does not. For another example, let us consider the ferromagnetism. The atoms in the ferromagnet interact through a spin-spin interaction $\mathrm{H}=-\sum_{\mathrm{i}, \mathrm{j}} \mathrm{J}_{\mathrm{i}, \mathrm{j}} \mathrm{S}_{\mathrm{i}} \mathrm{S}_{\mathrm{j}}$ which is a scalar and therefore invariant under rotations. However, in the ground state all the spins (within a domain) are arranged in a particular direction and not rotationally invariant. Thus, the system possesses a symmetry (rotation symmetry) but the ground state is not invariant under that symmetry.

Around 1960, Nambu and Goldstone realized the significance of SSB in condensed matter physics and Nambu in particular speculated on its application to particle physics. In 1964, Higgs pointed out that the consequences of spontaneous symmetry breaking in gauge theories which were different from those in non-gauge theories. Weinberg and Salam, building on earlier work of Glashow, then applied Higgs ideas to an $\mathrm{SU}(2)_{\mathrm{L}} \times \mathrm{U}(1)_{\mathrm{Y}}$ gauge theory which described weak and electromagnetic interactions together. SSB plays vital role in mass generation of fundamental particles.

The Higgs mechanism [12] allows generating particle masses while preserving the gauge symmetry of EW interactions [15]. This mechanism postulates the existence of a doublet of a complex scalar field which can be represented as [7]:

$$
\Phi=\left(\begin{array}{c}
\operatorname{Re} \Phi^{+}+i \operatorname{Im} \Phi^{+} \\
\operatorname{Re} \Phi^{0}+i \operatorname{Im} \Phi^{0}
\end{array}\right)
$$

and this scalar field is associated with a potential

$$
\mathrm{V}=\mu^{2} \Phi^{+} \Phi+\lambda\left(\Phi^{+} \Phi\right)^{2}
$$

which is invariant under the transformation of electroweak symmetry group $\mathrm{SU}(2)_{\mathrm{L}} \times \mathrm{U}(1)_{\mathrm{Y}}$. In the above equation $\mu^{2}$ stands for mass term of the field $\Phi$ and $\lambda(>0)$ stands for coupling constant of its self interaction.
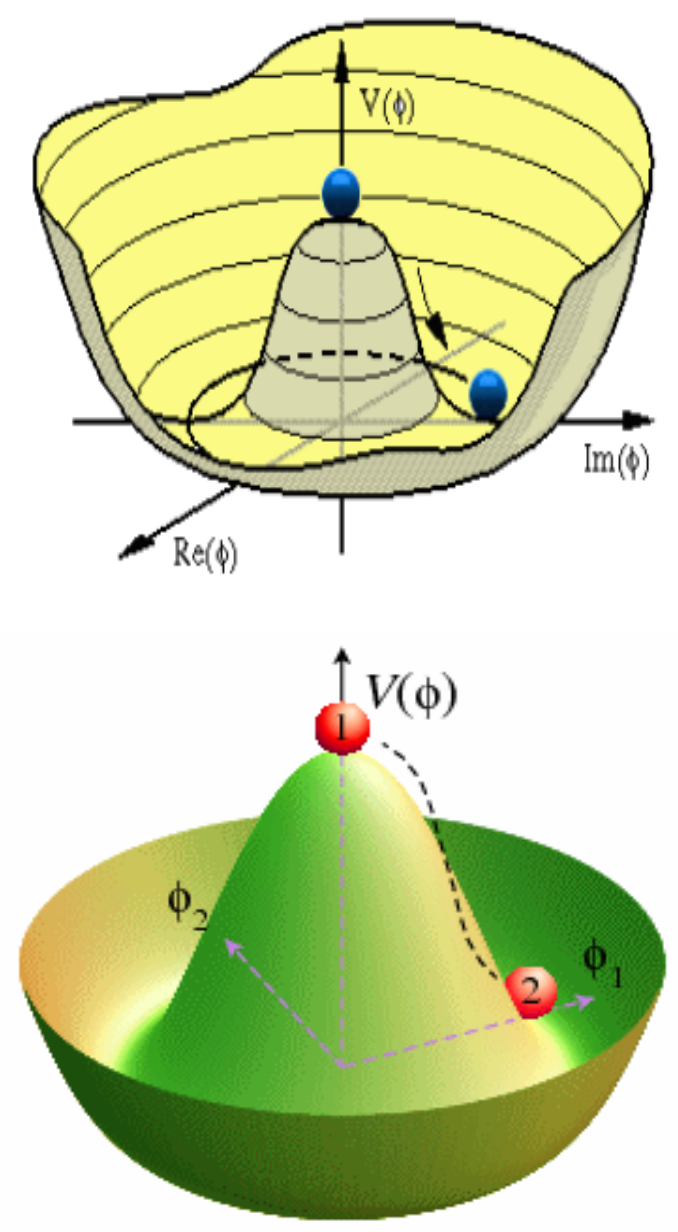

Figure 1. The potential of the scalar field $\Phi$ with its minimum at the value $v$ of the field.

For the positive values of $\mu^{2}$, the potential $\mathrm{V}(\Phi)$ has the form of an inverted bell where minimum field value corresponds to the state of vacuum with minimum potential energy which should be stable. If $\mu^{2}$ is negative, then the situation changes. In this case, the potential V $(\Phi)$ acquires the shape of a bottom of champagne bottle as shown in Figure-1 [3]. The vertical axis is the magnitude of potential energy and the horizontal axes represent the magnitude of the Higgs field. When the Higgs field is zero (point 1) then the potential energy is a maximum. The minimum of the potential is not reached for a zero value of the field $\Phi$ whereas it reaches a non-zero minimum value $\sqrt{\frac{-\mu^{2}}{\lambda}}$, which is called as vacuum expectation value of the field $\Phi$. 
The spontaneous symmetry breaking has the consequence of creating a non-zero Higgs field everywhere in space. The presence of a non-zero Higgs field in the universe interacting with particles results in most of them acquiring mass. When SSB occurs, the potential energy is reduced to one of the minimum points around the valley (point 2 ) and hence the Higgs field becomes non-zero. The radius of the bottom of the valley is related to the magnitude of the Higgs field which will be calculated from the strength of the weak force. Hence, it is the spontaneous breaking of EW symmetry which generates the masses of the vector bosons in a gauge invariant method.

Introduction of SSB in the SM needs to add a new doublet of quantum fields and each of these quantum fields is complex with a real and an imaginary part. As a result four new degrees of freedom (dof) are added up in the theory. Hence, we have four additional scalar fields corresponding to each dof. Out of the four dof of the complex doublet field $\Phi$, three have been absorbed by three gauge bosons of the weak interaction $\left(\mathrm{W}^{ \pm}, \mathrm{Z}^{0}\right)$. Initially these spin-1 fields were massless. But with two transverse components of dof these spin-1 fields achieves an additional dof corresponding to their longitudinal component which was the characteristic signature of their mass [7]. Later it was concluded that three of the dof of the field $\Phi$ have been absorbed by the three gauge bosons $\mathrm{W}^{ \pm}, \mathrm{Z}^{0}$ to acquire mass and the residual dof corresponds to a new particle, now known as Higgs boson [13]. After the confirmation of Higgs boson, the SM appears to be the right theory at the electroweak scale with all its parameters and fields.

\section{Unique status of Higgs boson}

The Higgs boson holds a unique status in the table of elementary particles due to its special properties which we are going to discuss now. The Higgs boson is a new type of particle, unlike other matter particles with spin-1/2 and gauge bosons of spin- 1 ; it is the only spin- 0 elementary particle at present. It is a boson but it does not mediate gauge interactions like other gauge bosons. The Higgs particle interacts with elementary particles proportional to their masses i.e. the particle acquires more mass if it couples more strongly with Higgs boson. For example, it couples most strongly to the top quark, the heaviest particle in the particle zoo of the SM. The Higgs boson does not directly couple with photons and gluons, they are massless. But coupling can be induced indirectly through quantum fluctuations. Gluons do carry colour charge. The Higgs boson can emit pair of heavy particles such as top quarks, which are reabsorbed by the Higgs particle immediately. These intermediate particles are called virtual particles, as these particles do not have the right mass and can only exist because of the Heisenberg uncertainty principle. Within the period of emission and absorption of these virtual particles photons and gluons are also generated. So, Higgs-photon-photon and Higgsgluons-gluons coupling can be induced in an indirect way known as quantum fluctuation. The Higgs boson is self-interacting and the magnitude of triple and quadratic self- interaction are proportional to $\mathrm{M}_{\mathrm{H}}$ (mass of Higgs boson). But the mass of Higgs boson $\mathrm{M}_{\mathrm{H}}$ is not determined in the SM. $\mathrm{M}_{\mathrm{H}}$ is not completely free as it has some experimental and theoretical constraints. Experimental constraints are due to the limitation on the operational energy bound of colliders (e.g. LHC and Tevatron). Analysis of electroweak high-precision data [16] allows an upper bound for $\mathrm{M}_{\mathrm{H}}$ around $152 \mathrm{GeV}$ at $95 \%$ C.L. In the SM, there is an upper bound for the mass of Higgs boson, $\mathrm{M}_{\mathrm{H}}<1 \mathrm{TeV}$ [17].

\section{Production and Decay of Higgs Boson}

The Higgs boson is an elusive particle. It can be created inside particle accelerators having high operational energy bound but its direct presence cannot be ascertained. It is studied only through its various decay modes and productions. The basic principle of production of Higgs particle is the transformation of beam K.E. into mass (energy) of the boson [8]. As the Higgs boson generates the mass of elementary particle, and it strongly couples with heavier particles, hence it can be produced via a radiation from heavy particles such as massive gauge boson $\left(\mathrm{W}^{ \pm}\right.$and $\left.\mathrm{Z}^{0}\right)$ or top quark $(\mathrm{t})$. Feynman diagrams for the production of Higgs particle at the Large Hadron Collider (LHC) is shown in Figure-2 [7,14]. Feynman diagrams are pictorial representations of the mathematical expressions governing the behavior of subatomic particles. This scheme was first introduced in 1948 by Richard Feynman, a famous American Physicist and Nobel Laureate. Generally interactions of subatomic particles are complex and difficult to understand. Feynman diagrams represent a simple visualization of the interaction.

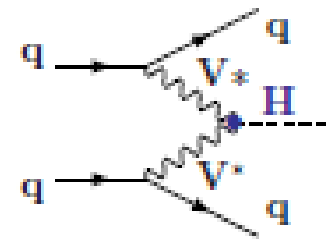

(a)

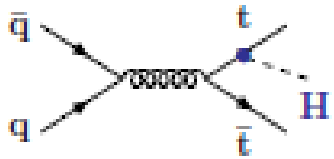

(b)

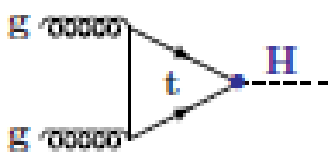

(c)

Figure 2. Feynman diagrams for the dominant Higgs production mechanisms at the LHC

Since a top quark has a mass of $173.5 \mathrm{GeV}$ and mass of proton is $0.938 \mathrm{GeV}$, top quarks are not generally found inside the beam particles. But it is possible to create virtual top quarks in several interaction processes. One particular example is shown in Figure-2(c). Here two gluons from two colliding beam of protons produces a pair of top quark or 
antiquark. These intermediate quarks pair again annihilates to give a Higgs boson. There are several other methods exist for the production and decay of Higgs boson which depend on the beam energy of colliding particles. An important factor in these observations is to reduce the background noise. The background noise is the event other than the required interaction which is unavoidable. The production of Higgs boson is also accessible at lower energy in the Fermilab Tevatron, in which production occurs through virtual quarks associated with $\mathrm{W}^{ \pm}$and $\mathrm{Z}^{0}$ boson. These associated productions are useful as the background noise is much smaller. In the production process, the Higgs boson has a short life time $\approx 10^{-22} \mathrm{sec}$. Four Higgs boson production mechanisms are currently being probed at LHC [18]:

(A) Fermionic production

(I) Gluon Fusion

(II) Top Quark Pair Associated Production

(B) Bosonic production

(III) Vector Boson Fusion

(IV) Associated Production with a Boson

The Higgs boson decays to several heavy particles which are consistent with conservation of energy. Figure-3 [8] shows the decay fractions of Higgs boson corresponding to different mass range of Higgs boson. Not all decay modes are equally informative or easy to detect. The $\mathrm{H} \rightarrow \gamma \gamma$ and $\mathrm{H} \rightarrow \mathrm{ZZ} \rightarrow 41$ (with $\mathrm{l}=\mathrm{e}, \mu$ ), channels play a special role due to the excellent mass resolution of the reconstructed diphoton and four-lepton final states respectively. Whereas the $\mathrm{H} \rightarrow$ WW $\rightarrow$ vv channel provides high sensitivity but has relatively poor mass resolution due to the presence of neutrinos in the final state. The mass of Higgs boson at the LHC is obtained by using the channels $\mathrm{gg} \rightarrow \mathrm{H} \rightarrow \gamma \gamma$ and gg $\rightarrow \mathrm{ZZ} \rightarrow 41$ only.

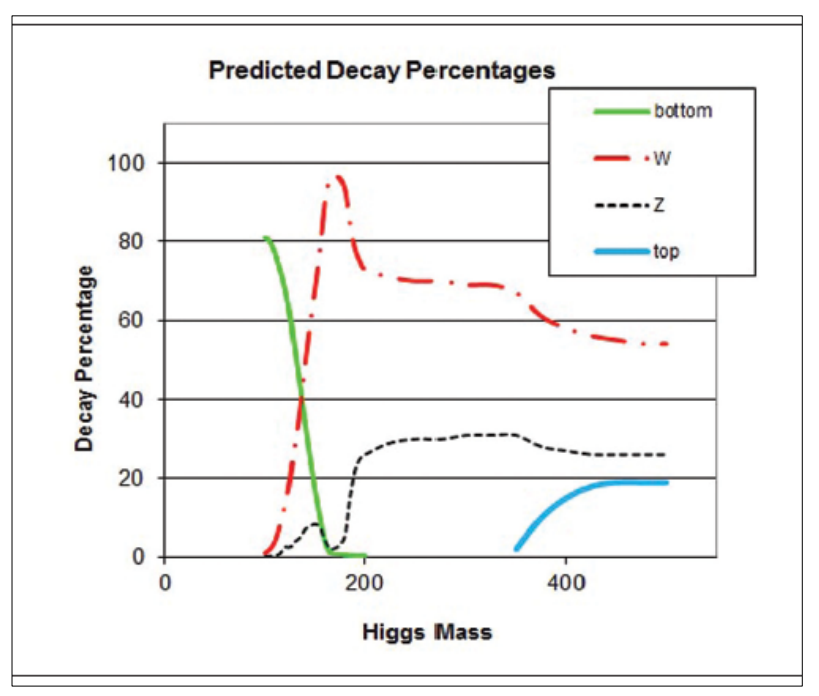

Figure 3. The decay fractions of the Higgs boson are completely specified by the theory for each value of the mass of the Higgs boson. This plot shows the range of possible decay modes.

\section{Further Discussions}

In July 2012, the CMS and ATLAS collaborations at the
LHC discovered independently a new particle with mass $125-126 \mathrm{GeV}$ and named 'Higgs like particle' [19,20]. On $14^{\text {th }}$ March, 2013, at the 'Moriond' conference in Italy, the LHC has confirmed the discovery of Higgs boson [21]. The discovery of Higgs boson was a giant step in the history of science, justifying the 2013 Nobel Prize in physics to Francois Englert and Peter Higgs. The Higgs boson is an excitation of the Higgs field which permeates all space. After the big bang, when this field cooled along with the rest of the universe, it underwent a condensation. An excitation of the Higgs condensate requires smashing particles together at high energies that could only be attained at the world's most powerful accelerator laboratories - the Tevatron near Chicago and the LHC near Geneva. The Higgs boson has been confirmed but whether it is the SM Higgs boson or a particle beyond the SM is not confirmed yet. Physics beyond the SM refers to the theoretical developments needed to explain the deficiencies of the SM such as the neutrino oscillations, matter-antimatter asymmetry of the universe and the nature of dark matter and dark energy. Another problem is that, the SM does not take into account the gravitational force. The gravitational force is based on the Einstein's general theory of relativity. This theory is based on the principles of classical mechanics and not of quantum mechanics. But other forces in nature obey the rules of quantum mechanics. That is why the SM is inconsistent with general theory of relativity. Theories beyond SM include Minimal Supersymmetric Standard Model (MSSM) or entirely novel explanations like string theory/M theory. Supersymmetry introduces a fermionic (bosonic) partner for every boson (fermion) differing by half a unit of spin quantum number. This partner is called superpartner (sparticle) of the original particle. Hence, every SM particle has a superpartner that has yet to be discovered. The MSSM is a minimal extension to the SM that realizes supersymmetry, although non-minimal extensions do exist. The supersymmetric extension of SM predicts the existence of different Higgs particle. The MSSM predicts the smallest number of Higgs boson i.e. five. These are: two CP even neutral Higgs boson (h and $\mathrm{H}$ ), a CP odd neutral Higgs boson (A) and two charged Higgs particle $\left(\mathrm{H}^{+}\right.$and $\left.\mathrm{H}^{-}\right)$. The lightest neutral Higgs particle is $\mathrm{h}$ and it has same properties as the SM Higgs particle with mass below $140 \mathrm{GeV}$.

Another important property of the new particle is its intrinsic spin. In the SM it is a spin-0 particle. The LHC physicists have already said that it is a boson. So it may have spin $0,1,2$ or some other integer [22]. Since this new particle decays into pairs of photons (photons are spin-1 bosons), its spin can not be 1 . Again physicists do not have unrealistic theories involving bosons with spin greater than 2. Hence, it is required to confirm whether it is a spin-2 or a spin-0 particle! Furthermore, the mass of Higgs boson is not determined in the SM. There are several experimental as well as theoretical constraints on the determination of its mass. The LEP experiments [23] predicted the lower limit on the mass of the Higgs boson as $114.4 \mathrm{GeV}$. There is an upper bound for the mass of Higgs boson, $\mathrm{M}_{\mathrm{H}}<1 \mathrm{TeV}$ [17]. The 
mass of the SM Higgs boson is $125.3 \pm 0.4$ (stat.) \pm 0.5 (syst.) $\mathrm{GeV}$ (CMS) [20] and $126.0 \pm 0.4$ (stat) \pm 0.4 (syst.) GeV (ATLAS) [24]. At the Tevatron, the CDF and DO Collaborations have observed the mass of Higgs boson in the range 115 to $140 \mathrm{GeV}$ [25]. Their observed signals are consistent with the SM Higgs boson with a mass of $125 \mathrm{GeV}$. More interestingly, Sahoo et al. [26] have predicted the mass of Higgs boson as $\mathrm{M}_{\mathrm{H}} \approx 120 \mathrm{GeV}$ in a flavor-independent model.

The Higgs discovery demonstrates the unity of physics. In the SM, it bridges the matter particles on the left and force particles on the right providing mass to both kinds of particles. Some of the problems in cosmology are related to the Higgs boson. The recent Planck mission's [27,28] observations demonstrate that our universe consists of $4.9 \%$ ordinary atoms (baryonic matter), $\sim 26.8 \%$ dark matter and $68.3 \%$ dark energy. Jackson et al. [29] have suggested that the Higgs boson might interact with weakly interacting massive particles of dark matter. The Higgs field does not directly couple to the quanta of light (photons) and it generates mass. Similarly, dark matter does not interact with the electromagnetic force and is responsible for the discrepancy between the apparent observed mass of the universe and its actual mass. Hence, it is expected that there is relation between Higgs boson and dark matter. The Higgs boson could be coupled to the particle that constitutes all or part of the dark matter in the universe [30]. Hertzberg [31] has predicted a relation between the Higgs mass and the abundance of dark matter. His theoretical result is good agreement with current data. He has predicted the mass of Higgs boson as $125.7 \pm 0.6 \mathrm{GeV}$ which consistent with the recent prediction at the LHC i.e. 125-126 GeV $[19,20]$. Recently Huang [32] has discussed phase dynamics of the Higgs field on a cosmic scale. According to him, the Higgs field fills all space. On microscopic scale, it gives mass to elementary particles. On macroscopic scale, it flows like a superfluid, due to phase variations. On cosmic scale, it makes the universe a superfluid. The energy of this cosmic superfluid can be identified with dark energy, and fluctuations of the superfluid density lead to dark matter. It is also claimed [33] that graphene would help physicists to probe the Higgs boson's secrets. When we compress graphene it ripples and displays a sort of symmetry breaking and we know that Higgs mechanism explains symmetry breaking. Thus, the Higgs boson can act as a bridge between particle physics as well as condensed matter physics and cosmology [34].

The discovery of Higgs boson is expected to be a portal for the investigation of new physics beyond the SM which includes supersymmetry, extra dimension, strongly interacting particles and many more. The LHC is now shut down mode for maintenance and refurbishment of some parts of the accelerator so that it will be able to reach its full design energy $(14 \mathrm{TeV})$. It is expected to resume operation in the spring of 2015. We are eagerly waiting for detailed measurement of Higgs properties and some other discoveries at the LHC in the coming decades.

\section{Acknowledgments}

We thank the referee for suggesting valuable improvements of our manuscript. S. Sahoo acknowledges NIT Durgapur for providing financial supports "Research Initiation Grant" through office order No. NITD/Regis/OR/25 dated 31 ${ }^{\text {st }}$ March, 2014.

\section{REFERENCES}

[1] D. Griffiths, Introduction to Elementary Particles, John Wiley and Sons, Singapore (1987).

[2] D. Banerjee and S. Sahoo, Physics Education, 29(3), Article Number: 04, pp. 1-7 (2013); G. Rajasekaran, Resonance, 17, 956 (2012).

[3] K. E. Johansson and P.M. Watkins, Phys. Edu. 48, 105 (2013).

[4] S. Sahoo, Eur. J. Phys. 30, 901 (2009); S. Sahoo, Physics Education, 22, 85 (2005).

[5] R. M. Godbole, Fundamental particles and their interactions, in Horizons of Physics Vol. I Ed. A. W. Joshi, Wiley Eastern Ltd., Delhi (1989).

[6] D. Lincoln, Understanding the Universe: From Quarks to the Cosmos (Revised) World Scientific Press, Singapore (2012).

[7] A. Djouadi, AIP Conf. Proc. 1444, 45 (2012).

[8] D. Lincoln, The Physics Teacher, 50, doi: 10.1119/1.4745683 (2012).

[9] B. Schumm, Deep Down Things: The Breathtaking Beauty of Particle Physics, Johns Hopkins University Press, Baltimore (2004).

[10] S. Sahoo and M. Goswami, IAPT Bulletin, 4(2), 39 (2012); S. Sahoo and R. K. Agarwalla, IAPT Bulletin, 25, 293 (2008).

[11] P. Dirac, Proc. Roy. Soc. Lond. A114, 243 (1927); P. Jordan and W. Pauli, Z. Phys. 47, 151 (1928); W. Heisenberg and W. Pauli, Z. Phys. 56, 1 (1929); S. Tomonaga, Progr. Theor. Phys. 1, 27 (1946); J. Schwinger, Phys. Rev. 73, 416 (1948); R. Feynman, Phys. Rev. 76, 749 (1949).

[12] F. Englert and R. Brout, Phys. Rev. Lett. 13, 321 (1964); P. W. Higgs, Phys. Rev. Lett. 13, 508 (1964); G. S. Guralinik, C. R. Hagens and T. W. B. Kibble, Phys. Rev. Lett. 13, 585 (1964).

[13] P. Ghosh, S. Sadhu and S. Sahoo, ARPN Journal of Science and Technology, 4(2), 83 (2014); S. Sahoo and S. Sadhu, Emerging Science, 5 (2013).

[14] A. Djouadi, Phys. Rep. 457, 1-216 (2008) [hep-ph/0503172].

[15] S. Glashow, Nucl. Phys. 22, 579 (1961); S. Weinberg, Phys. Rev. Lett. 19, 1264 (1967); A. Salam, in "Elementary Particle Theory", Stockholm (1969) p. 367; G.'t Hooft and M. Veltman, Nucl. Phys. B44, 189-213 (1972).

[16] ALEPH, CDF, D0, DELPHI, L3, OPAL, SLD Collabs., the LEP Electroweak Working Group, the Tevatron Electroweak Working Group, and the SLD Electroweak and Heavy Flavour Groups, Precision electroweak measurements and 
constraints on the standard model, CERN PH-EP-2010-095, http://lepewwg.web.cern.ch/LEPEWWG/plots/winter2012/, arXiv:1012.2367 (2010), http://cdsweb.cern.ch/record/13137 16.

[17] T. S. Virdee, Int. J. Mod. Phys. A, 29(9), 1430019 (2014).

[18] S. Meola, arXiv: 1310.4146 [hep-ex] (2013); G. Landsberg, arXiv: 1310.5705 [hep-ex] (2013).

[19] G. Aad et al. (ATLAS Collaboration), Phys. Lett. B 716, 1 (2012).

[20] S. Chatrchyan et al. (CMS Collaboration), Phys. Lett. B 716, 30 (2012) [arXiv: 1207.7235 [hep-ex]].

[21] H. Dreiner, Nature Physics, 9, 268(2013).

[22] M. Chalmers, Nature, 488, 572 (2012).

[23] ALEPH, DELPHI, L3, OPAL Collabs. and LEP Working Group for Higgs Boson Searches, Phys. Lett. B 565, 61 (2003).

[24] S. M. Consonni (on behalf of the ATLAS Collaboration), arXiv:1305.3315 [hep-ex] (2013).
[25] T. Aaltonen et al. (CDF and DO Collaboration), Phys. Rev. D, 88, 052014 (2013) [arXiv:1303.6346 [hep-ex]].

[26] S. Sahoo, A. K. Meikap and L. Maharana, Mod. Phys. Lett. A 21, 275 (2006)

[27] P. Chen, arXiv:1310.1107 [hep-ph] (2013).

[28] P. A. R. Ade et al. [Planck Collaboration], arXiv:1303.5062 [astro-ph.CO] (2013).

[29] C. B. Jackson, S. Servant, G. Shaughnessy, T. M. P. Tait and M. Taoso, JCAP, 04, 004 (2010) [arXiv: 0912.0004 [hep-ph]].

[30] C. A. Solans, arXiv:1407.7377 [hep-ex] (2014).

[31] M. P. Hertzberg, arXiv:1210.3624 [hep-ph] (2012).

[32] K. Huang, Int. J. Mod. Phys. A, 29(9), 1430022 (2014).

[33] http://www.searchthenetnow.com/graphene-could-help-phys icists-probe-the-higgs-bosons-secrets

[34] S. Sahoo and B. K. Sahoo, ARPN Journal of Science and Technology, 2(10), 912 (2012). 